\title{
La relación hispano-brasileña en la Unión Ibérica a través de los documentos conservados en instituciones españolas
}

\author{
Aurora Cuevas-Cerveró \\ Universidad Complutense de Madrid, Facultad de Ciencias de la Documentación, Madrid, España \\ macuevas@ucm.es \\ https://orcid.org/0000-0002-0928-0403 \\ Ignacio Miró-Charbonnier \\ Universidad Alfonso X El Sabio, Madrid, España \\ jmiro@ucm.es \\ https://orcid.org/0000-0002-4057-0716
}

DOI: https://doi.org/10.26512/rici.v13.n2.2020.30874

Recebido/Recibido/Received: 2019-12-15

Aceitado/Aceptado/Accepted: 2020-04-14

Resumen: Presentamos los primeros resultados de un proyecto de investigación que tiene como objetivo la identificación y difusión de valiosos documentos históricos relativos al periodo de la Unión lbérica (1580-1640) que se conservan en las instituciones documentales españolas. Este proyecto aporta luz a una época poco conocida de la historia común de Brasil y España y pretende estrechar los vínculos entre ambos países. La metodología para la identificación de los documentos parte de una revisión bibliográfica que guiará la selección de fuentes en los archivos y bibliotecas de España. Una vez identificados, los documentos seleccionados serán organizados en función de una taxonomía de áreas temáticas establecida a partir de la investigación de los fondos más relevantes de las instituciones citadas. En una primera etapa se realizará la identificación, selección y eventual digitalización de la documentación. Una segunda etapa procurará acercar a la ciudadanía y a los investigadores una selección de los documentos más relevantes a través de diferentes iniciativas entre las que cabe destacar: exposiciones virtuales y presenciales, en España y Brasil; publicaciones científicas y divulgativas y la creación de un portal web donde se enlace y describa una selección de las obras digitalizadas. De esta forma ese importante legado documental quedará más accesible tanto para la comunidad investigadora como para la ciudadanía.

Palabras-clave: Unión Ibérica. legado documental. proyecto de investigación. Archivos. Bibliotecas. Digitalización.

The Brazilian-Hispanic relationship in the Iberian Union through documents preserved in Spanish institutions

Abstract: We present the first results of a research project that aims at identifying and disseminating valuable historical documents related to the period of the Iberian Union (1580-1640) that are preserved in Spanish documentary institutions. This project sheds light on a little-known era in the shared history of Brazil and Spain and intends to strengthen ties between the two countries. The first methodological step for the identification of documents has been a bibliographic review that will help in the identification and selection of sources in Spanish archives and libraries. Once the selected documents are identified, they will be classified after a taxonomy of thematic areas resulting from research of the most relevant holdings. 
In a first stage the identification, selection and digitization of the documentation will be carried out. A second stage should bring to citizens and researchers alike a selection of the most relevant documents; this will be possible through various initiatives, notably: virtual and physical exhibitions, in Spain and Brazil; scientific and informative publications; and the creation of a web portal where a selection of digitized works will be linked and described. Through all those means; this important documentary legacy will be made much more accessible to the research community as well as to every citizen.

Keywords: Iberian Union. documentary legacy. research project. Archives. Libraries. digitization.

\section{A relação hispano-brasileira na União Ibérica através dos documentos preservados nas instituições espanholas}

Resumo: Apresentamos os primeiros resultados de um projeto de pesquisa que visa identificar e divulgar os documentos históricos valiosos relacionados ao período da União Ibérica (1580-1640), mantidos em instituições documentais espanholas. Este projeto lança luz sobre uma era pouco conhecida na história comum do Brasil e da Espanha e visa fortalecer os laços entre os dois países. A metodologia para a identificação de documentos parte de uma revisão bibliográfica que orientará a seleção de fontes nos arquivos e bibliotecas da Espanha. Uma vez identificados, os documentos selecionados serão organizados de acordo com uma taxonomia de áreas temáticas estabelecidas a partir da investigação dos fundos mais relevantes das instituições mencionadas. Numa primeira etapa, será realizada a identificação, seleção e eventual digitalização da documentação. Uma segunda etapa procurará aproximar cidadãos e pesquisadores de uma seleção dos documentos mais relevantes por meio de diferentes iniciativas, incluindo: exposições virtuais e presenciais na Espanha e no Brasil; publicações científicas e informativas e a criação de um portal na web em que uma seleção de obras digitalizadas é vinculada e descrita. Dessa maneira, esse importante legado documental ficará mais acessível à comunidade de pesquisa e aos cidadãos.

Palavras-chave: União Europeia. legado documental. projeto de pesquisa. arquivos. bibliotecas. digitalização.

\section{Introducción}

El Imperio portugués, del que Brasil formaba parte, fue gobernado por la monarquía española desde el reinado de Felipe II, y se separó en el reinado de Felipe IV. Este periodo (1580 y 1640) denominado "Filipino" o "periodo de la Unión Ibérica", produjo una gran cantidad de documentación, que se encuentra ubicada en diferentes instituciones españolas; las más importantes son el Archivo de Simancas y el Archivo General de Indias, pero también la Biblioteca Nacional de España, el Archivo Histórico Nacional, y otras organizaciones como el Museo del Prado o el Archivo y Biblioteca del Ministerio de Asuntos Exteriores.

Los documentos preservados en esas instituciones son apenas conocidos y han sido poco divulgados a pesar de su relevancia para la historia de Brasil en relación con España. Ejemplos a destacar, de entre los acontecimientos más importantes acaecidos durante ese periodo de la Unión Ibérica pueden ser la introducción en Brasil de las Ordenações filipinas que rigieron el derecho civil brasileño hasta la elaboración del Código Civil de 1916; las invasiones holandesas en el Nordeste; y el inicio de la expansión hacia el Oeste del territorio del Brasil Colonial. 
La historiografía portuguesa, brasileña y española no se ha interesado especialmente en este periodo, entre otras razones por la relación peculiar que se produjo entre España y Portugal a partir de 1640, y por el distanciamiento que tuvo lugar entre Brasil y Portugal desde 1822 (TORRES MEGIANI, SANTOS PÉREZ y VANDERLEI SILVA, 2014). Sin embargo, en las últimas décadas han aumentado los historiadores interesados en las cuestiones relativas a este periodo, destacando entre ellos Bouza Álvarez (2000), Labrador Arroyo (2009), Torres Megiani, Santos Pérez y Vanderlei Silva (2014) y Valladares $(2000,2012)$.

Al consultar la literatura especializada que trata específicamente de los documentos de los siglos XVI y XVII referidos al periodo en que los países de España y Portugal estuvieron bajo una misma corona, hemos encontrado publicaciones relevantes sobre las fuentes documentales existentes; y lo mismo resulta aplicable al caso concreto de las relaciones que, durante esa "Unión Ibérica", mantuvieron España y la colonia, hasta entonces portuguesa, de Brasil. Entre esas publicaciones especializadas figuran de manera destacada las de autores como Cabral de Mello Neto (1966), Dos Santos (1993), Santaella (1990, 2000), López Gómez y García Miraz (2000), González Martínez (2002), Olarán Mújica (2008), García López (2009), y Vainfas (2017).

Sin embargo, y a pesar de la importancia indudable que esas publicaciones tienen para los investigadores especializados en la materia, no parece ser que los resultados de sus trabajos hayan producido un efecto señalado en amplios sectores del público general, sea en Brasil o en España. Por el contrario, se detecta en ese público un notorio desconocimiento en cuanto a casi todo lo relacionado con la etapa histórica común a los dos países; e incluso se ignora con frecuencia el hecho mismo de que España, Portugal y Brasil estuvieron regidos bajo una misma corona durante más de medio siglo.

A los hechos anteriores, que ya justificarían por sí solos un proyecto como el que aquí exponemos, se añade el terrible suceso, acaecido el 2 de septiembre de 2018, del incendio del Museo Nacional de Brasil en Rio de Janeiro, que causó la destrucción de unos 20 millones de piezas. A partir de ese momento, las acciones de la comunidad internacional dirigidas a localizar y digitalizar los documentos relacionados con la historia de Brasil que se encuentren custodiados fuera de ese país han revestido una importancia adicional de primer orden.

Por todo lo antes expuesto, hemos considerado del mayor interés planificar un proyecto de investigación que favorezca de manera significativa el conocimiento de la etapa histórica mencionada. Ese proyecto tomará en consideración las fuentes ya citadas, sumará nuestra propia indagación, y diseñará los medios para una mejor y más amplia divulgación de los principales documentos que en ella se produjeron. En particular nos propondremos que nuestros resultados aporten a las instituciones y personas interesadas, particularmente en Brasil 
y España, un acceso más directo a información multimedia que trate de documentos especialmente relevantes para la memoria histórica de los dos países.

Hemos escogido para nuestro proyecto el siguiente título: "La historia de Brasil en los Documentos conservados en España, identificación y difusión". El equipo de investigación está compuesto por investigadores de la Universidad Complutense de Madrid, Universidad de Brasilia y Universidad Federal de Paraíba, y será financiado por la Embajada de Brasil en España y por el Grupo de investigación "Información, Biblioteca y Sociedad" de la Universidad Complutense de Madrid.
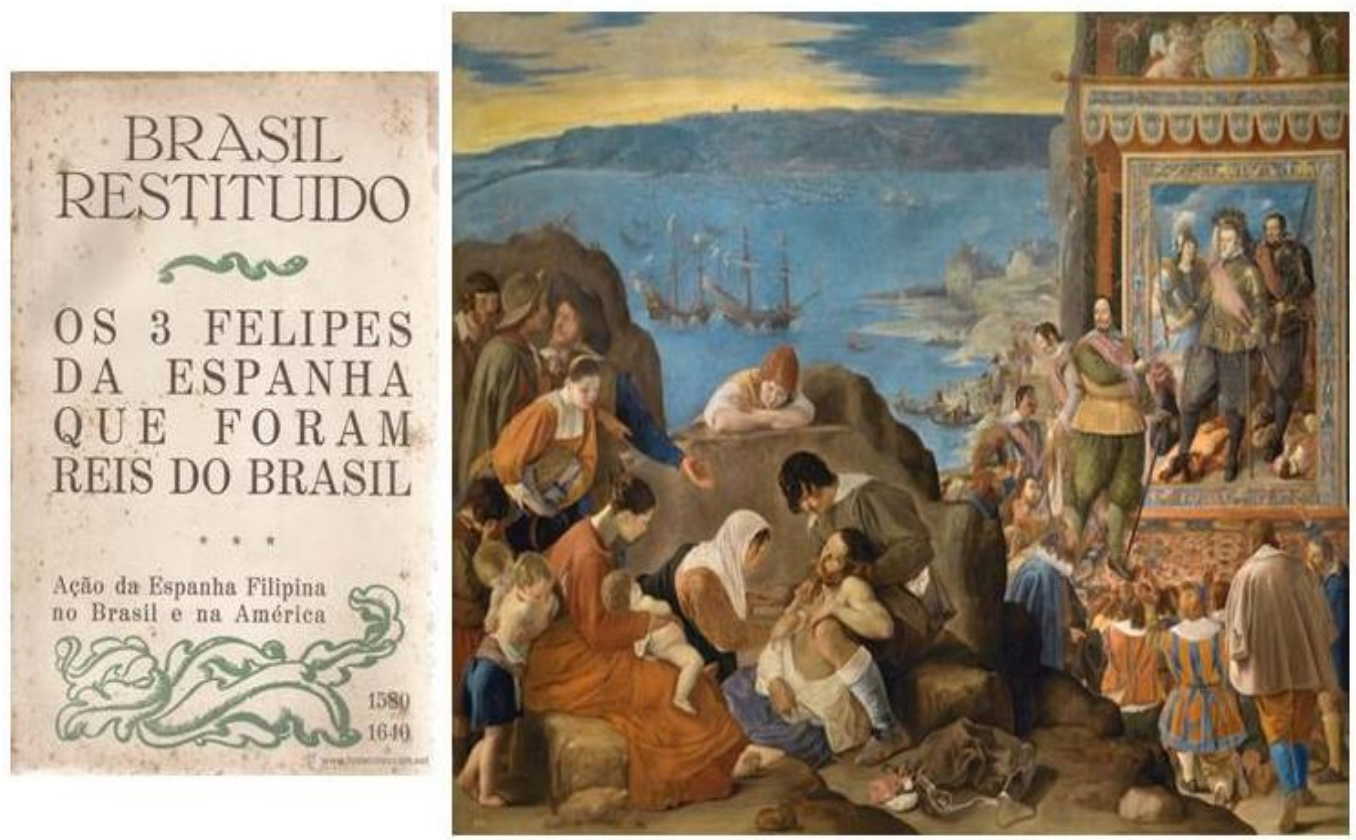

Ilustración 1. Dos muestras de obras de arte que trataron de la Unión Ibérica: una reedición de la comedia "El Brasil restituido" (Lope de Vega, 1625) y el cuadro "La recuperación de Bahía de Todos los Santos" (Maíno, 1635)

\section{Metodología}

Los objetivos concretos que han sido formulados para el proyecto han procurado atender a las cinco fases previstas. Estas fases, y sus correspondientes objetivos de investigación, pueden describirse como sigue:

A. En primer lugar se seleccionará, de entre los documentos identificados en archivos y otras instituciones españolas, una selección de aquellos que se

\footnotetext{
${ }^{1}$ El original autógrafo de esa obra de Lope de Vega, fechado en Madrid a 23 de octubre de 1625, se conserva en la Biblioteca Pública de Nueva York, SASB - Manuscripts \& Archives Rm 328 (MssCol 3151), procedente de la ya desaparecida Lenox Library, de la misma ciudad; de él posee la Biblioteca Nacional de España una copia manual y otra fotostática. El cuadro de Fray Juan Bautista Maíno se encuentra en el Museo del Prado, Madrid.
} 
presenten como especialmente relevantes para el conocimiento de lo que la etapa citada aportó a uno y otro país. No se pretende elaborar una enumeración exhaustiva de todos los documentos existentes, sino destacar los más representativos para cada una de las áreas temáticas previstas. En esa selección podrán figurar tanto manuscritos como documentos de imprenta.

B. Una vez efectuada esa selección, habrá que ofrecer descripciones y /o transcripciones de esos documentos. Esas descripciones y /o transcripciones se crearán o partiendo de cero o bien recopilando, cuando se disponga de ellas, las que previamente hayan preparado sobre esos documentos sus respectivas instituciones custodias, o los investigadores externos que los hayan estudiado.

C. A continuación será necesario unificar esas descripciones y /o transcripciones, adaptando cada una de ellas de manera que pueda quedar integrada en un conjunto lo más homogéneo posible.

D. Adicionalmente, se deberá impulsar la digitalización de los documentos originales, o bien, cuando se cuente ya con copias digitales de los mismos que puedan ser divulgadas, habrá que actuar de manera similar a como se ha indicado para las descripciones y /o transcripciones documentales.

E. Y, por último, habrá que diseñar y llevar a cabo estrategias de difusión de los documentos, a través de sus descripciones así como de las copias digitales de aquellos, para que consigan llegar a un público no-especialista, gracias a la puesta en práctica de enfoques divulgativos especialmente adecuados para ese fin.

\subsection{Selección de documentos en función de áreas y categorías temáticas}

Una primera revisión de las fuentes documentales ya identificadas para la época en cuestión muestra no solamente una importante abundancia documental, sino también una no menos grande variedad temática. El proyecto previsto debe abordar esa variedad de temas tratados de manera que la haga atractiva al público potencial, y le permita ver la importancia que el periodo estudiado tuvo en las distintas manifestaciones y actividades de las sociedades involucradas por ese contacto decisivo que se produjo entonces entre España y Brasil.

Se hace necesario, pues, organizar esa variedad de temas de manera que quede articulada de forma coherente, y no caiga por el contrario en una mera acumulación de asuntos, por atractivos que éstos pudieran resultar para el público esperado.

En una primera fase del proyecto, la investigación se centrará en el estudio de los fondos custodiados en una selección de centros: el Archivo General de Simancas, en la provincia de Valladolid; la Biblioteca Nacional de España; el Archivo Histórico Nacional; y el Archivo y Biblioteca del Ministerio de Asuntos Exteriores. Una segunda fase del proyecto está prevista que sea iniciada un año más tarde, y su investigación se dirigiría a indagar en los fondos custodiados en otros centros, destacando entre ellos el Archivo General de Indias, en la ciudad de Sevilla.

\subsection{Criterios de la selección proyectada}


Para efectuar la organización temática mencionada, y tras las consultas bibliográficas necesarias y varias consultas directas a expertos en la materia, nos hemos basado en clasificaciones tomadas de la literatura especializada sobre el tema, para adaptarlas luego a nuestros propios objetivos.

En concreto, hemos tomado como referente principal la clasificación formulada por Pedro López Gómez (Universidad de A Coruña) y María del Mar García Miraz (Archivo del Reino de Galicia), en el artículo publicado en la Revista de Indias el año 2000 y titulado "Fuentes archivísticas para la Historia del Brasil en España (siglos XV-XVII)".

En la Introducción a dicho artículo, al hablar de las fuentes documentales e instrumentos descriptivos disponibles, sus autores mencionan una sucesión de temas o asuntos en los que las instituciones relevantes en dicho momento histórico tenían competencias: "conocimiento, exploración, colonización, ocupación y defensa militar, administración, explotación económica, penetración religiosa y culturización del Brasil”.

Esa enumeración nos resultaba a la vez muy significativa desde el punto de vista de la investigación histórica, por venir de autores que habían profundizado en el examen de muchos de los principales archivos custodios de información sobre el periodo de la Unión Ibérica; pero se nos hacía demasiado plana desde el punto de vista de la ciencia documental, y más concretamente teniendo en cuenta los propósitos clasificatorios propios de esta ciencia. En consecuencia, hemos formulado una taxonomía que contiene un conjunto propio de grandes áreas temáticas. Ese conjunto nace por tanto de la enumeración citada de López Gómez y García Miraz, pero una vez contrastada con los cuerpos documentales que íbamos viendo referidos en los demás estudios consultados sobre la Unión Ibérica, y más particularmente sobre su rama brasileña.

De esa manera, hemos establecido un primer estado de clasificación temática, consistente en ocho grandes áreas del conocimiento; éstas se han subdivido a su vez en un número variable de categorías, veinticuatro en total. Y decimos "un primer estado" porque somos conscientes de que, según avance la investigación y lo requieran los documentos encontrados y seleccionados, será probablemente necesario ir adaptando a ese cuerpo documental la estructura prevista, en una medida que aún desconocemos.

Confiamos en poder conseguir una mejor organización de los contenidos de esos documentos, organización que deberá atender a la mejor divulgación posible de los fondos. Esta preocupación por la divulgación presidirá las decisiones metodológicas a tomar, sin detrimento del rigor investigador que deba ser puesto en ellas.

\section{3 Áreas y categorías previstas:}


Las ocho áreas temáticas previstas serán las siguientes, referidas de manera abreviada:

1. Cultura / Arte

2. Conocimiento / Ciencias Naturales

3. Penetración religiosa / Misiones

4. Exploración, colonización y ocupación

5. Explotación económica / comercio

6. Sociedad

7. Historia /Relatos

8. Derecho / Legislación

En cuanto a las divisiones o categorías temáticas a las que inicialmente se atenderá dentro de esas áreas, serán como indicamos en la tabla 1.

Tabla 1

\begin{tabular}{|c|c|}
\hline 1. Cultura / Arte & $\begin{array}{ll}\text { - } & \text { Literatura } \\
\text { - } & \text { Artes plásticas } \\
\text { - } & \text { Música } \\
\text { - } & \text { Arquitectura / Urbanismo } \\
\text { - } & \text { Lenguas autóctonas }\end{array}$ \\
\hline 2. Conocimiento / Ciencias Naturales & $\begin{array}{ll}\text { - } & \text { Botánica } \\
\text { - } & \text { Zoología } \\
\text { - } & \text { Otras Ciencias Naturales } \\
\end{array}$ \\
\hline 3. Penetración religiosa / Misiones & $\begin{array}{ll}\text { - } & \text { Jesuitas } \\
\text { - } & \text { Franciscanos } \\
\text { - Otras órdenes (Capuchinos, } \\
\text { etc.) }\end{array}$ \\
\hline $\begin{array}{l}\text { 4. Exploración, colonización y } \\
\text { ocupación }\end{array}$ & $\begin{array}{ll}\text { - } & \text { Expediciones terrestres y } \\
& \text { marítimas / expansión } \\
\text { - } & \text { Defensa militar / Fortificaciones } \\
\text { - } & \text { Piratería }\end{array}$ \\
\hline 5. Explotación económica / Comercio & $\begin{array}{ll} & \text { Madera: palo de Brasil, etc. } \\
\text { - } & \text { Alimentos: caña de azúcar, etc. } \\
\text { - } & \text { Otros artículos comerciales }\end{array}$ \\
\hline 6. Sociedad & $\begin{array}{ll}\cdot & \text { Etnografía y vida cotidiana } \\
\text { - } & \text { Esclavitud } \\
\text { - } & \text { Mestizaje } \\
\end{array}$ \\
\hline 7. Historia / Relatos & $\begin{array}{ll} & \text { Relatos europeos } \\
\text { - } & \text { Relatos indígenas }\end{array}$ \\
\hline 8. Derecho / Legislación & $\begin{array}{ll}\text { - } & \text { Ordenaciones filipinas } \\
\text { - } & \text { Otras regulaciones }\end{array}$ \\
\hline
\end{tabular}

Taxonomía para las áreas temáticas del proyecto. (Fuente: elaboración propia)

Los documentos que resulten seleccionados para cada una de las categorías temáticas previstas deberán tener carácter representativo; por ello, habrá que someterlos a un proceso que los lleve desde el terreno de los investigadores especializados hasta el ámbito del gran 
público. En ese proceso, hemos previsto dos fases principales, cada una de las cuales deberá atender a varios objetivos específicos.

Primera fase:

- Digitalización de los documentos seleccionados para cada área y categoría.

- Transcripción y/ o Descripción de los documentos que lo requieran.

- Estudio histórico de cada área y categoría, para una mejor contextualización de los documentos seleccionados.

- Análisis histórico/documental detallado de los más relevantes.

- Compilación bibliográfica del periodo de la Unión Ibérica relativa a los aspectos tratados en las categorías previstas.

Segunda fase:

- Difusión de la documentación de archivo que haya sido seleccionada, clasificada y transcrita, mediante:

- Un portal web creado expresamente para la difusión prevista;

- Comunicaciones en congresos y seminarios;

- Publicaciones en revistas especializadas; y

- Exposiciones virtuales y presenciales dirigidas a un público general, no especializado; y donde podrán tener cabida tanto los documentos originales que puedan ser prestados por las instituciones custodias, como las copias digitales de aquellos que no cumplan lo anterior.

\section{3 llustración de los primeros resultados}

Hemos querido ilustrar mediante un ejemplo el tipo de resultados concretos que el proyecto espera obtener, en cuanto a los documentos que cabría seleccionar y describir con particular detalle, de cara a su posterior difusión (cf. Ilustración 1).

Se trata de una unidad documental simple, cuya signatura antigua era DIVERSOSDOCUMENTOS_INDIAS, N.334, lo que nos indica que estuvo anteriormente conservada en el Archivo de Indias (Sevilla); actualmente se encuentra bajo la custodia del Archivo Histórico Nacional de España (AHN) en Madrid, con el código de referencia ES.28079.AHN//DIVERSOSCOLECCIONES,26,N.76.

Dentro del cuadro de clasificación del Archivo Histórico Nacional se ubica en "COLECCIONES" (5), dentro de la sección "DOCUMENTOS TEXTUALES" (5.1), formando parte de la "Colección Documentos de Indias"2.

\footnotetext{
${ }^{2}$ Fuente: http://pares.mcu.es/ParesBusquedas20/catalogo/description/1339551?nm
} 
Ilustración 2. Unidad documental de ejemplo.

Fuente: Archivo Histórico Nacional de España (Madrid)

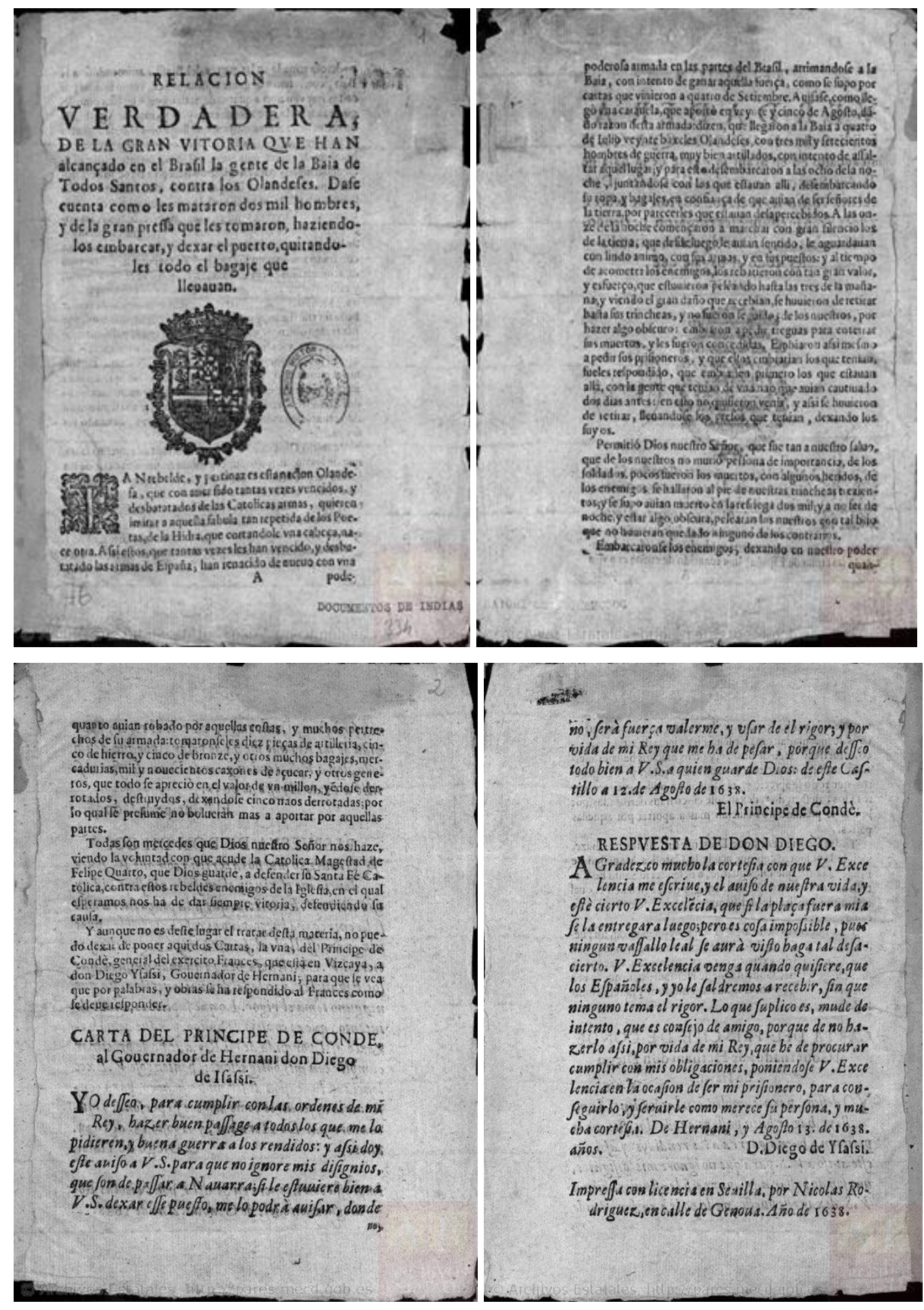

El asunto tratado en esta colección es la famosa batalla contra los holandeses, tema presente en otros muchos documentos preservados en distintos archivos españoles. 
La ocupación militar por parte de Holanda, y el saqueo posterior, del enclave ibérico de "Salvador de Todos los Santos", hoy llamado "Salvador de Bahía", acaeció el 9 de mayo de 1624. Cuando en la corte española se tuvo noticia de este hecho, se reunió la mayor fuerza naval disponible que partió del puerto de Cádiz el 14 de enero de 1625. Llegaron a Cabo Verde el 4 de febrero para que se les uniera una escuadra portuguesa. En total sumaron 52 buques y 12.463 soldados españoles, portugueses y napolitanos bajo el mando del español Don Fadrique de Toledo y Osorio, Capitán General de la Armada. El 29 de marzo llegaron a Bahía, y asediaron a los holandeses hasta que se rindieron un mes más tarde. El 1 de mayo de 1625, los holandeses aceptaron la capitulación que Don Fadrique les ofreció, quedando restituido así el dominio hispano-portugués en Bahía.

La unidad documental que hemos elegido en relación con esos acontecimientos se compone de varios elementos:

1ํ) Una narración del hecho histórico mencionado bajo el título "Relación verdadera de la gran victoria que han alcanzado en el Brasil la gente de la Bahía de Todos los Santos contra los Holandeses" (Relación verdadera de la gran vitoria que han alcançado en el Brasil la gente de la Bahia de Todos Santos contra los Olandeses).

2ㅇ) Dos cartas que no tienen relación con el hecho histórico anteriormente narrado (el autor dice textualmente al terminar la narración: "Y aunque no es de este lugar tratar de esta materia, no puedo dejar de poner aquí dos cartas..."), pero que se ofrecen como muestra de la caballerosidad y gallardía que los españoles empleaban con sus enemigos en los conflictos bélicos.

a) La del francés Príncipe de Condè al Gobernador de Hernani ('Carta del Príncipe de Conde, al Gouernador de Hernani don Diego de Isassi'), fechada el 12 de agosto de 1638, por tanto más de diez años después del acontecimiento bélico que la motivaba; $y$

b) La de don Diego de Isasi en respuesta a la carta anterior ('Respuesta de Don Diego'), y fechada en Hernani tan sólo un día más tarde, el 13 agosto de 1638.

Esta unidad documental fue impresa en agosto de ese mismo año de 1638, en Sevilla, por Nicolás Rodríguez, quien acompañó a los textos citados de diversas ilustraciones e iniciales grabadas, como señala la referencia bibliográfica correspondiente, elaborada por el AHN.

Según la clasificación ya comentada de áreas y categorías temáticas que se ha previsto para el proyecto, cabría considerar a la colección documental en cuestión como altamente representativa del área y categoría siguientes:

- Área Cuarta (de las ocho previstas inicialmente en el proyecto): Explotación, colonización, ocupación 
- Categoría Segunda (de las tres previstas dentro del área Cuarta): Defensa militar.

\section{A modo de conclusión}

El proyecto acaba de comenzar su andadura pero ya ha despertado gran interés institucional tanto en Brasil como en España, pues promueve el entendimiento y la cooperación entre los dos países. La aspiración principal de esta investigación es la divulgación de sus resultados y no solo entre historiadores, archiveros o investigadores, sino también entre un público general, entre la ciudadanía. Esta difusión se efectuará a través de varios medios, entre ellos -y muy especialmente- un portal web públicamente accesible, que pondrá todo este acervo documental seleccionado, clasificado temáticamente, y comentado- a disposición de todos, y que, al mismo tiempo, cumplirá una función de conservación y preservación de ese patrimonio documental, mediante su digitalización.

Esta primera etapa la investigación se ha centrado en los aspectos metodológicos, muy importantes pues de ellos depende que las clasificaciones y descripciones estén bien fundamentadas, de manera que sean rigurosas a la vez que puedan llegar al gran público.

En cuanto a los temas que serán contemplados, entendemos que la literatura, el arte, el urbanismo o la vida cotidiana son manifestaciones culturales muy importantes que acercan a los pueblos y colaboran a un buen entendimiento; por ello, les daremos la misma importancia que a los asuntos administrativos o políticos, que suelen ser el principal objeto de estudio.

Pretendemos con ello que este proyecto tenga al mismo tiempo un cariz científico y cultural; que aporte luz a un periodo poco conocido de la historia común de Brasil y España; y que, con todo ello, contribuya a fortalecer la memoria histórica de España y Brasil, estrechando así los vínculos entre ellos.

\section{Referencias:}

BOUZA ALVAREZ, Fernando J. Portugal no tempo dos Filipes: política, cultura, representações (1580-1668). Lisboa: Cosmos, 2000. (História, 34) ISBN 972-762-195-3

GARCÍA LÓPEZ, M. B. Fuentes para la Historia colonial de Brasil en los archivos españoles. Nuevo Mundo / Mundos Nuevos, Guía del investigador americanista. 2009. Recuperado de: https://journals.openedition.org/nuevomundo/56980 [Consulta: 01/03/2020]

GONZÁLEZ MARTíNEZ, E. E. Guía de fuentes manuscritas para la Historia de Brasil conservadas en España. Madrid: Fundación Mapfre Tavera, 2002. ISBN 978-8484790273

LABRADOR ARROYO, Félix. La Casa Real en Portugal (1580-1621). Madrid: Polifemo Ediciones,2009. 
LÓPEZ GÓMEZ, P.; GARCÍA MIRAZ, M. M. Fuentes archivísticas para la Historia de Brasil en España (siglos XV-XVII). Revista de Indias, v. 60, n. 218, p. 135-179, 2000.

MARTÍNEZ ORTIZ, J. Documentos manuscritos y obras varias impresas referentes a la historia del Brasil, existentes en Valencia (España). Valencia: Instituto de Estudios Americanistas, Institución Alfonso el Magnánimo: Diputación Provincial de Valencia: Caja de Ahorros y Monte de Piedad de Valencia, 1969.

MEGIANI, Ana Paula Torres; SANTOS PÉREZ, José Manuel; SILVA, Kalina Vanderlei. O Brasil na Monarquia Hispânica (1580-1668). Novas interpretações. São Paulo: Humanitas, 2014.

MELLO NETO, João Cabral de; RODRIGUES, José Honório. O Arquivo das Índias e o Brasil: documentos para a história do Brasil existentes no Arquivo das Índias de Sevilha, Rio de Janeiro: Ministério das Relações Exteriores, Comissionado de Textos de História do Brasil, 1966.

ORARÁN MÚGICA, Maria Inés. El Consejo de Portugal en el Archivo General de Simancas; Fuente para la historia del Brasil colonial. In: CONGRESSO DA ASBRAP, ASSOCIAÇÃO BRASILEIRA DE PESQUISADORES DE HISTÓRIA E GENEALOGIA, 1., 2008. Actas. Diálogos entre História e Genealogia", São Paulo, Brasil, 2 de agosto de 2008.

SANTOS, Ricardo Evaristo. El Brasil filipino: 60 años de presencia española en Brasil (1580-1640). Madrid: Mapfre, 1993. ISBN 978-8471005946

SANTOS PÉREZ, J. M. La conquista y colonización de Maranhão-Grão Pará: el gran proyecto de la Monarquía Hispánica para la Amazonia brasileña (1580-1640). Revista de Estudios Brasileños, v. 6, n. 11, p. 33-47, 2019. Disponible en: https://revistas.usal.es/index.php/23864540/article/view/reb20196113347

SOUTO MAIOR, P. Nos arquivos de Hespahna. Relação dos manuscriptos que interessam ao Brasil. Revista do Instituto Histórico e Geográfico Brasileiro, v. 80, p. 7-207; y v. 81, p. 9-288, 1918.

SOUZA JÚNIOR, A. O período filipino da História do Brasil nos arquivos espanhóis. Revista do Instituto de Geografia e História Militar do Brasil, v. XLIII, n. 56, p. 103-114, 1968.

STELLA SANTAELLA, Roseli. Brasil durante el gobierno español: 1580-1640. Madrid: Fundación Mapfre Tavera, 2000.

STELLA SANTAELLA, Roseli. Catálogo dos documentos relativos ao Brasil existentes no Arquivo Geral de Simancas. Espanha, sec. XVI e XVII. São Paulo: Departamento de História Faculdade de Ciências e Letras Cruzeiro do Sul, 1990.

VAINFAS, R. Tempo dos Filipes no Brasil colonial: enfoques historiográficos, possibilidades de investigação. Revista Maracanan, n. 16, p. 14-33, Jan./junho 2017. Disponible en: https://www.e-publicacoes.ueri.br/index.php/maracanan/article/view/27118/19588

VALLADARES RAMÍREZ, Rafael Brasil: de la unión de Coronas a la crisis de Sacramento (15801680). Acuarela de Brasil, 500 años después: seis ensayos sobre la realidad histórica y económica brasileña: II Coloquio Internacional de Historia de América, Salamanca 10-12 de noviembre de 1999. Salamanca: Ediciones Universidad de Salamanca, 2000. 
VALLADARES RAMÍREZ, Rafael. No somos tan grandes como imaginábamos. Historia global y Monarquía Hispánica. UNED. Espacio, Tiempo y Forma. Serie IV, Historia Moderna, t. 25, 2012. Disponible en: http://revistas.uned.es/index.php/ETFIV/article/view/11948/11351

\section{Agradecimientos}

A la Embajada de Brasil en Madrid, España (Contrato de investigación 12, 2019) y al Consulado de España en Río de Janeiro, Brasil, por su apoyo y empuje en la realización de esta investigación.

A la Dra. Ana García-Herranz, por su valiosa colaboración en el proyecto de investigación y en este artículo. 